\title{
Bumblebee size polymorphism and worker response to queen pheromone
}

Queen pheromones are chemical signals produced by reproductive individuals in social insect colonies. In many species they are key to the maintenance of reproductive division of labor, with workers beginning to reproduce individually once the queen pheromone disappears. Recently, a queen pheromone that negatively affects worker fecundity was discovered in the bumblebee Bombus terrestris, presenting an exciting opportunity for comparisons with analogous queen pheromones in independently-evolved eusocial lineages such as honey bees, ants, wasps and termites. I set out to replicate this discovery and verify its reproducibility. Using blind, controlled experiments, I found that $n$-pentacosane $\left(C_{25}\right)$ does indeed negatively affect worker ovary development. Moreover, the pheromone affects both large and small workers, and applies to workers from large, mature colonies as well as young colonies. Given that $\mathrm{C}_{25}$ is readily available and that bumblebees are popular study organisms, I hope that this replication will encourage other researchers to tackle the many research questions enabled by the discovery of a queen pheromone.[b] 


\section{Bumblebee size polymorphism and}

2 worker response to queen

3 pheromone

4 Short title: Bumblebee queen pheromone

\section{Luke Holman}

6 luke.holman@anu.edu.au

7 Centre of Excellence in Biological Interactions,

8 Division of Ecology, Evolution \& Genetics,

9 Research School of Biology,

10 Australian National University,

11 Canberra, ACT 0200, Australia.

12 Word count: 2034 in main text. 
13 Keywords: Bombus terrestris, Eusociality, Fertility signal, Reproducible

14 research, Social insects

\section{Abstract}

16 Queen pheromones are chemical signals produced by reproductive

17 individuals in social insect colonies. In many species they are key to the

18 maintenance of reproductive division of labor, with workers beginning to

19 reproduce individually once the queen pheromone disappears. Recently, a

20 queen pheromone that negatively affects worker fecundity was discovered in

21 the bumblebee Bombus terrestris, presenting an exciting opportunity for

22 comparisons with analogous queen pheromones in independently-evolved

23 eusocial lineages such as honey bees, ants, wasps and termites. I set out to

24 replicate this discovery and verify its reproducibility. Using blind, controlled

25 experiments, I found that $n$-pentacosane $\left(C_{25}\right)$ does indeed negatively affect

26 worker ovary development. Moreover, the pheromone affects both large and

27 small workers, and applies to workers from large, mature colonies as well as

28 young colonies. Given that $C_{25}$ is readily available and that bumblebees are

29 popular study organisms, I hope that this replication will encourage other

30 researchers to tackle the many research questions enabled by the discovery

31 of a queen pheromone. 


\section{Introduction}

33 Queen pheromones are chemical signals produced by queens (or other fertile

34 females) to communicate with conspecifics, typically other colony members

35 such as workers. They have been implicated in a great many worker

36 responses to queens, including a negative effect on worker ovary

37 development or reproduction

38 (Hoover et al. 2003; Matsuura et al. 2010; Holman et al. 2010; Van Oystaeyen

39 et al. 2014), aggregation around the queen

40 (Keeling et al. 2003), differential behavioral development

41 (Hoover et al. 2003; Vergoz et al. 2007; Matsuura et al. 2010; Holman et al.

42 2010; Van Oystaeyen et al. 2014), and a wealth of upstream genetic and 43 physiological effects

44 (Kaatz et al. 1992; Keeling et al. 2003; Grozinger et al. 2003; Malka et al. 45 2014). Until recently, experimental evidence linking specific queen-produced 46 chemicals to worker responses was largely limited to the well-studied 47 honeybee (Apis mellifera), though there is a wealth of non-experimental 48 evidence that queen pheromones are widespread (reviewed in Kocher and 49 Grozinger 2011; Van Oystaeyen et al. 2014). In the last four years, additional 50 queen pheromones were experimentally isolated in a few ant species (Smith 
51 et al. 2009; Holman et al. 2010; Smith et al. 2012; Holman et al. 2013; Van

52 Oystaeyen et al. 2014), a wasp and a bumblebee (Van Oystaeyen et al.

53 2014), and a termite

54 (Matsuura et al. 2010; Matsuura 2012). It is thus an exciting time to study

55 queen pheromones, since their discovery in these diverse, independently

56 evolved eusocial lineages poses many new questions.

57 As well as pressing on with queen pheromone research, e.g. by 58 unraveling their full diversity, documenting their effects, and determining 59 their evolutionary significance, I believe it is worthwhile to replicate and 60 validate initial reports of newly discovered pheromones. Replication of

61 empirical work remains somewhat rare throughout the life sciences, likely 62 because novel results are perceived as more valuable (loannidis 2005; Kelly 63 2006). Experiments can generate false positives for many reasons, and the 64 false positive rate is probably substantially higher than the $5 \%$ implied by the 65 familiar $p<0.05$ threshold due to the combined influence of many forms of 66 experimental and statistical bias (loannidis 2005; Simonsohn et al. 2014), 67 making replication essential to scientific progress.

68 I set out to replicate a recent finding that the cuticular hydrocarbon $n$ 69 pentacosane (hereafter $\mathrm{C}_{25}$ ) is a queen pheromone that causes sterility in 70 workers of the bumblebee Bombus terrestris. Van Oystaeyen et al. (2014) 71 exposed queenless bumblebee workers to this hydrocarbon or one of four 72 different esters 


\section{PeerJ Reviewing Manuscript}

73 (these five compounds were previously found to be characteristic of queens

74 and fertile workers; Sramkova et al. 2008), then dissected them to determine

75 whether the workers' ovaries had remained comparatively inactive relative to

76 those of control workers (which were expected to develop their ovaries after

77 the removal of their queen). The four esters had no effect, but $C_{25}$

78 significantly reduced worker ovary development relative to the control.

79 Although I was an author on this study, I did not participate in the design or

80 execution of the bumblebee experiment, and I deliberately remained ignorant

81 of the methods used beyond what is written in the paper. Thus, a replication

82 by me is likely to be as similar to the original study as a replication by any

83 other researcher. Due to practical constraints, I opted to test only whether $C_{25}$

84 affects worker ovaries, and not to re-test whether the four esters have no 85 effect.

86 Repeating the experiment also provided the opportunity to gather

87 additional data and try revised methods. In order to test whether previous

88 findings generalize to different colony stages, I used large (c. 300 workers),

89 mature bumblebee colonies, unlike Oystaeyen et al. (2014), who used small,

90 developing colonies containing about 20 workers each. Testing the effects of

91 queen pheromones in mature colonies is interesting, because large

92 bumblebee colonies reach the so-called "competition point", at which many

93 workers begin to reproduce, often while the queen is still present (Van Doorn

94 and Heringa 1986; Duchateau and Velthuis 1989; Alaux et al. 2004). This

95 implies that the response of workers to queen pheromone may decline

96 following the competition point. Additionally, bumblebee colonies naturally 


\section{PeerJ Reviewing Manuscript}

97 produce workers which vary as much as 10-fold in body size, likely because

98 different sized workers are better at different tasks (reviewed in Couvillon et

99 al. 2010), which affects their fecundity (Foster et al. 2004). By recording body

100 size in my experiment, I was able to test whether the effects of $C_{25}$ differ for

101 large (highly fecund) and small (weakly fecund) workers. Next, I did not use

102 the same ovary scoring system as van Oystaeyen et al. (2014), since I found

103 it difficult to use during preliminary trials (in particular, I could not reliably 104 identify "regressed" ovaries, if any were indeed present in my sample). 105 Instead, I simply counted developing oocytes. Finally, I used a lower dose of 106 pheromone. van Oystaeyen et al. (2014) applied $467 \mu \mathrm{g}$ of $\mathrm{C}_{25}$ per day to each 107 colony, which they estimated to be approximately twice the amount present 108 on the cuticle of a mature $B$. terrestris queen. To test whether $C_{25}$ is also 109 efficacious at lower doses (which might more closely mimic the traces of $C_{25}$

110 deposited by the queen on the nest substrate as she moves around, though 111 this remains to be measured), I arbitrarily selected a dose of $2 \mu \mathrm{g}$ per day, or

112 approximately $1 / 100^{\text {th }}$ of a queen equivalent. In sum, my study is a "partial 113 replication" (reviewed in Kelly 2006), since it replicates the majority of the 114 design of the original experiment, but studies a later colony stage, adds a 115 measure of worker size, uses a different dose of queen pheromone and an 116 alternative measure of worker ovarian development.

\section{Methods}

118 Ten queenright bumblebee colonies (worker number: $300 \pm 20$ ) were 119 obtained from Borregaard BioPlant (Denmark), and kept in the plastic cages 


\section{PeerJ Reviewing Manuscript}

120 provided by that company. These colonies had presumably passed the

121 competition point (or would soon do), since they had many more workers

122 than colonies in which worker egg laying has been observed previously (e.g.

123 Van Doorn and Heringa 1986). The colonies had constant access to sugar

124 water via a feeder and were given pollen ad libitum (the sugar feeder and

125 pollen were obtained from Borregaard BioPlant), and were kept at room

126 temperature. Feeding and pheromone application was performed under red

127 light.

128 I first removed the queens, using red light illumination to sort through

129 the colonies without anesthesia. Half of the colonies were randomly assigned

130 to the $C_{25}$ (286931, Sigma-Aldrich) treatment $\left(0.01 \mu \mathrm{g} \mu^{-1}\right.$ solution in hexane),

131 and half to the hexane-only control (HPLC-grade hexane was used

132 throughout; 34859, Sigma-Aldrich). Every 24h for 14 days, I added a total of

$133200 \mu$ l of hydrocarbon solution $\left(C_{25}\right.$ or hexane; i.e. $2 \mu \mathrm{g}$ of $C_{25}$ in the $C_{25}$

134 treatment) to the colony by pipetting it through the cage lids onto multiple

135 areas of the cotton wool that lined the nests, taking care to avoid the bees.

136 After 14 days, colonies were freeze-killed. I then dissected a randomly

137 selected sample of 50-51 workers per colony to determine ovary

138 development. This was accomplished by counting the number of developing

139 oocytes present in the ovaries. Prior to dissection, I scored workers as

140 "Small", "Medium", or "Large", based on whether I estimated them to be in

141 the lower, middle, or upper third of the size range for B. terrestris workers.

142 Dissections and size classifications were performed blind to treatment, and 143 workers were processed in small batches (c. 15) taken from a randomly 


\section{PeerJ Reviewing Manuscript}

144 chosen colony to prevent order effects biasing the results. Since size

145 classification was performed prior to dissection, it was blind with respect to 146 ovary status.

147 The oocyte count data contained many zeros (394/502 workers had no 148 oocytes in their ovaries), precluding the use of Poisson-based models. I

149 therefore analysed the data with a generalized linear mixed model (GLMM) 150 with negative binomial errors and colony as a random factor (using the 151 function glmer.nb in the Ime4 package for R).

\section{Results}

$153 \mathrm{C}_{25}$-treated workers had fewer oocytes in their ovaries than controls (GLMM: $\mathrm{z}$ $154=-3.04, \mathrm{p}=0.0024, \mathrm{n}=502$ ), and larger workers had more oocytes (Medium 155 vs Large workers: $z=-3.30, p=0.0010$; Medium vs Small workers: $z=3.63$, $156 \mathrm{p}=0.00029)$. There was no evidence that workers of different sizes 157 responded differentially to $C_{25}$ treatment (likelihood ratio test of models with 158 and without the Treatment $\times$ Size interaction: $p=0.75 ; \Delta \mathrm{AIC}=3.4$; the 159 interaction term was removed when estimating the main effects in the above 160 statistics). The random effect "colony" explained very little variation in 161 oocyte number (variance associated with colony: $1.4 \times 10^{-10}$, residual

162 variance: 0.55$)$, though I left the colony effect in the model in order to be 163 conservative (the results of a negative binomial generalized linear model 164 lacking colony were near-identical).

165 The negative effect of $\mathrm{C}_{25}$ treatment on oocytes number might 166 theoretically be explained by a higher frequency of larger-bodied (i.e. more 
167 fertile) workers being sampled in the hexane-treated colonies due to chance.

168 In fact, the frequency of "Large" workers was non-significantly higher in the

$169 \mathrm{C}_{25}$ treatment than in the hexane treatment (91 vs 65; binomial GLMM with

170 colony as a random factor: $\mathrm{z}=1.68, \mathrm{p}=0.093, \mathrm{n}=502)$. The sampled

171 workers in the hexane-treated colonies were "Medium"-sized more often than

172 in the $\mathrm{C}_{25}$-treated colonies (126 vs 105), but this difference was not

173 significant $(z=1.17, p=0.24)$. Thus, there was no evidence that a chance

174 overabundance of larger-bodied workers in the hexane treatment might

175 explain the observed negative effect of $\mathrm{C}_{25}$ on worker oocyte number.

\section{Discussion}

177 The study replicated Van Oystaeyen et al. (2014)'s finding that $C_{25}$ is a queen

178 pheromone that negatively affects ovarian development in $B$. terrestris

179 workers. Furthermore, I found that although larger workers were more

180 fecund, the effect of the pheromone appeared to be consistent across the

181 range of worker sizes.

182 Because Van Oystaeyen et al. (2014)'s experiment used young

183 bumblebee colonies with few workers while the present study used larger,

184 older colonies, the present results also provide some evidence that the effect

185 of queen pheromone is constant across colony life stages. In turn, this implies

186 that the en masse development of worker ovaries which occurs after the

187 "competition point" cannot be explained by a loss of sensitivity to queen

188 pheromone (since bees from both young and old colonies appear sensitive to

189 the pheromone). Alternative explanations for the onset of worker 
190 reproduction (e.g. Van Doorn and Heringa 1986), such as a reduction in the

191 quantity of pheromone produced by the ageing queen, the declining

192 frequency of queen contact per worker as the colony grows, or the

193 involvement of other signals and cues (e.g. an additional chemical signal, or

194 simply the frequency of worker-worker contacts), thus seem more likely given

195 the present results.

196 Previous work on bumblebees has suggested that workers signal their

197 fecundity and position in the colony's dominance hierarchy via their chemical

198 profile, following the competition point. The egg-laying "elite" workers are

199 highly active and aggressive (Van Doorn and Heringa 1986) and are

200 hypothesized to advertise their high fecundity to their nestmates, inducing

201 them to remain sterile or face reprisals in the form of aggression or

202 destruction of their eggs

203 (Amsalem et al. 2009). Given this evidence, and the fact that a constant dose

204 of pheromone might represent a proportionately higher dose for small

205 individuals, one might have expected the pheromone to have a stronger

206 effect on smaller workers. However, I found no evidence that the effect of $C_{25}$

207 on ovarian development is size dependent.

208 Given the strength of the evidence that $C_{25}$ is involved in regulating

209 reproductive division of labor in $B$. terrestris (namely two independent, blind

210 experiments), it is my hope that other research groups will begin

211 experimenting with this system. Unlike some of the other recently-discovered

212 queen pheromones 
213 (e.g. 3-MeC $\mathrm{M}_{31}$ in ants and n-butyl-n-butyrate and 2-methyl-1-butanol in

214 termites; Matsuura et al. 2010; Holman et al. 2013), this chemical does not

215 need to be synthesized to order: it can be easily purchased, making it just as

216 readily available as the well-studied honey bee queen pheromone. Though

217 currently unstudied, it is possible that $\mathrm{C}_{25}$ is as multi-functional as other

218 queen pheromones

219 (Le Conte and Hefetz 2008; Holman 2010; Kocher and Grozinger 2011;

220 Matsuura 2012), and the proximate mechanisms by which it affects worker

221 ovarian development have yet to be discovered.

222 Data sharing

223 The raw data are archived at Dryad (URL to be added here).

\section{Acknowledgements}

225 I am grateful to the Centre for Social Evolution, University of Copenhagen, for

226 hosting these experiments, and to the Finnish Academy of Sciences and the

227 Australian Research Council for funding.

\section{References}

229 Alaux, C., P. Jaisson, and A. Hefetz. 2004. Queen influence on worker

230 reproduction in bumblebees (Bombus terrestris) colonies. Insect. Soc.

231 51:287-293. 
232 Amsalem, E., R. Twele, W. Francke, and A. Hefetz. 2009. Reproductive 233 competition in the bumble-bee Bombus terrestris: do workers advertise 234 sterility? Proc. Roy. Soc. B 276:1295-1304.

235 Couvillon, M. J., J. M. Jandt, N. Duong, and A. Dornhaus. 2010. Ontogeny of 236 worker body size distribution in bumble bee (Bombus impatiens) colonies.

237 Ecol Entomol 35:424-435.

238 Duchateau, M. J., and H. Velthuis. 1989. Ovarian development and egg laying 239 in workers of Bombus terrestris. Entomol. Exper. Applic. 51:199-213.

240 Foster, R. L., A. Brunskill, D. Verdirame, and S. O'Donnell. 2004. Reproductive 241 physiology, dominance interactions, and division of labour among bumble 242 bee workers. Physiol. Entomol. 29:327-334.

243 Grozinger, C. M., N. M. Sharabash, C. W. Whitfield, and G. E. Robinson. 2003. 244 Pheromone-mediated gene expression in the honey bee brain. PNAS 245 100:14519-14525.

246 Holman, L. 2010. Queen pheromones: The chemical crown governing insect 247 social life. Commun Integr Biol 3:558-560.

248 Holman, L., C. G. Jørgensen, J. Nielsen, and P. d'Ettorre. 2010. Identification of 249 an ant queen pheromone regulating worker sterility. Proc. Roy. Soc. B 250 277:3793-3800.

251 Holman, L., R. Lanfear, and P. d'Ettorre. 2013. The evolution of queen 252 pheromones in the ant genus Lasius. J. Evol. Biol. 17:1549-1558.

253 Hoover, S. E. R., C. I. Keeling, M. L. Winston, and K. N. Slessor. 2003. The 254 effect of queen pheromones on worker honey bee ovary development.

255 Naturwissenschaften 90:477-480.

256 Ioannidis, J. P. A. 2005. Why most published research findings are false. PLoS 257 Med. 2:e124.

258 Kaatz, H.-H., H. Hildebrandt, and W. Engels. 1992. Primer effect of queen 259 pheromone on juvenile hormone biosynthesis in adult worker honey bees. J. 260 Comp. Physiol. B 162:588-592.

261 Keeling, C. I., K. N. Slessor, H. A. Higo, and M. L. Winston. 2003. New 262 components of the honey bee (Apis mellifera L.) queen retinue pheromone.

263 PNAS 100:4486-4491.

264 Kelly, C. D. 2006. Replicating empirical research in behavioral ecology: how 265 and why it should be done but rarely ever is. Q. Rev. Biol. 81:221-236.

266 Kocher, S. D., and C. M. Grozinger. 2011. Cooperation, conflict, and the 267 evolution of queen pheromones. J Chem Ecol 37:1263-1275. 
268 Le Conte, Y., and A. Hefetz. 2008. Primer pheromones in social Hymenoptera.

269 Annu. Rev. Entomol. 53:523-542.

270 Malka, O., E. L. Niño, C. M. Grozinger, and A. Hefetz. 2014. Genomic analysis

271 of the interactions between social environment and social communication

272 systems in honey bees (Apis mellifera). Insect Biochem. Mol. Biol. 47:36-45.

273 Matsuura, K. 2012. Multifunctional queen pheromone and maintenance of

274 reproductive harmony in termite colonies. J Chem Ecol 1-9.

275 Matsuura, K., C. Himuro, T. Yokoi, Y. Yamamoto, E. L. Vargo, and L. Keller.

276 2010. Identification of a pheromone regulating caste differentiation in

277 termites. PNAS 107:12963-12968.

278 Simonsohn, U., L. D. Nelson, and J. P. Simmons. 2014. P-curve: A key to the

279 file-drawer. Journal of Experimental Psychology 143:534-547.

280 Smith, A. A., B. Hölldobler, and J. Liebig. 2009. Cuticular hydrocarbons reliably 281 identify cheaters and allow enforcement of altruism in a social insect. Curr.

282 Biol. 19:78-81.

283 Smith, A. A., J. G. Millar, L. M. Hanks, and A. V. Suarez. 2012. Experimental

284 evidence that workers recognize reproductives through cuticular

285 hydrocarbons in the ant Odontomachus brunneus. Behav. Ecol. Sociobiol.

286 66:1267-1276.

287 Sramkova, A., C. Schulz, R. Twele, W. Francke, and M. Ayasse. 2008. Fertility

288 signals in the bumblebee Bombus terrestris (Hymenoptera: Apidae).

289 Naturwissenschaften 95:515-522.

290 Van Doorn, A., and J. Heringa. 1986. The ontogeny of a dominance hierarchy

291 in colonies of the Bumblebee Bombus terrestris (Hymenoptera, Apidae).

292 Insect. Soc. 33:3-25.

293 Van Oystaeyen, A., R. C. Oliveira, L. Holman, J. S. van Zweden, C. Romero, C. 294 A. Oi, P. d'Ettorre, M. Khalesi, J. Billen, F. Wäckers, J. G. Millar, and T.

295 Wenseleers. 2014. Conserved class of queen pheromones stops social insect 296 workers from reproducing. Science 343:287-290.

297 Vergoz, V., H. A. Schreurs, and A. R. Mercer. 2007. Queen pheromone blocks

298 aversive learning in young worker bees. Science 317:384-386. 


\section{Figure legend}

300 Figure 1: Treatment with $n$-pentacosane $\left(C_{25}\right)$ reduced the number of oocytes 301 in the ovaries of queenless workers, and there was a strong positive effect of 302 worker body size (note different $y$ axes). The effect of $C_{25}$ did not differ 303 significantly between Large, Medium and Small workers. The violin plots show 304 the kernel density estimate (i.e. estimated frequency: wide areas contain 305 more data), and the scatter plot shows the raw data. The $x$ coordinates of the 306 raw data are arbitrary: the points were randomly "jittered" horizontally to 307 make overlapping points visible. 


\section{Figure 1}

Figure 1 - Ovaries by treatment and size. If the EPS looks weird, try the supplementary PDF version, or ask me and I will send an SVG version.

Figure 1: Treatment with $n$-pentacosane $\left(\mathrm{C}_{25}\right)$ reduced the number of oocytes in the ovaries of queenless workers, and there was a strong positive effect of worker body size (note different $y$ axes). The effect of $\mathrm{C}_{25}$ did not differ significantly between Large, Medium and Small workers. The violin plots show the kernel density estimate (i.e. estimated frequency: wide areas contain more data), and the scatter plot shows the raw data. The $x$ coordinates of the raw data are arbitrary: the points were randomly "jittered" horizontally to make overlapping points visible. 

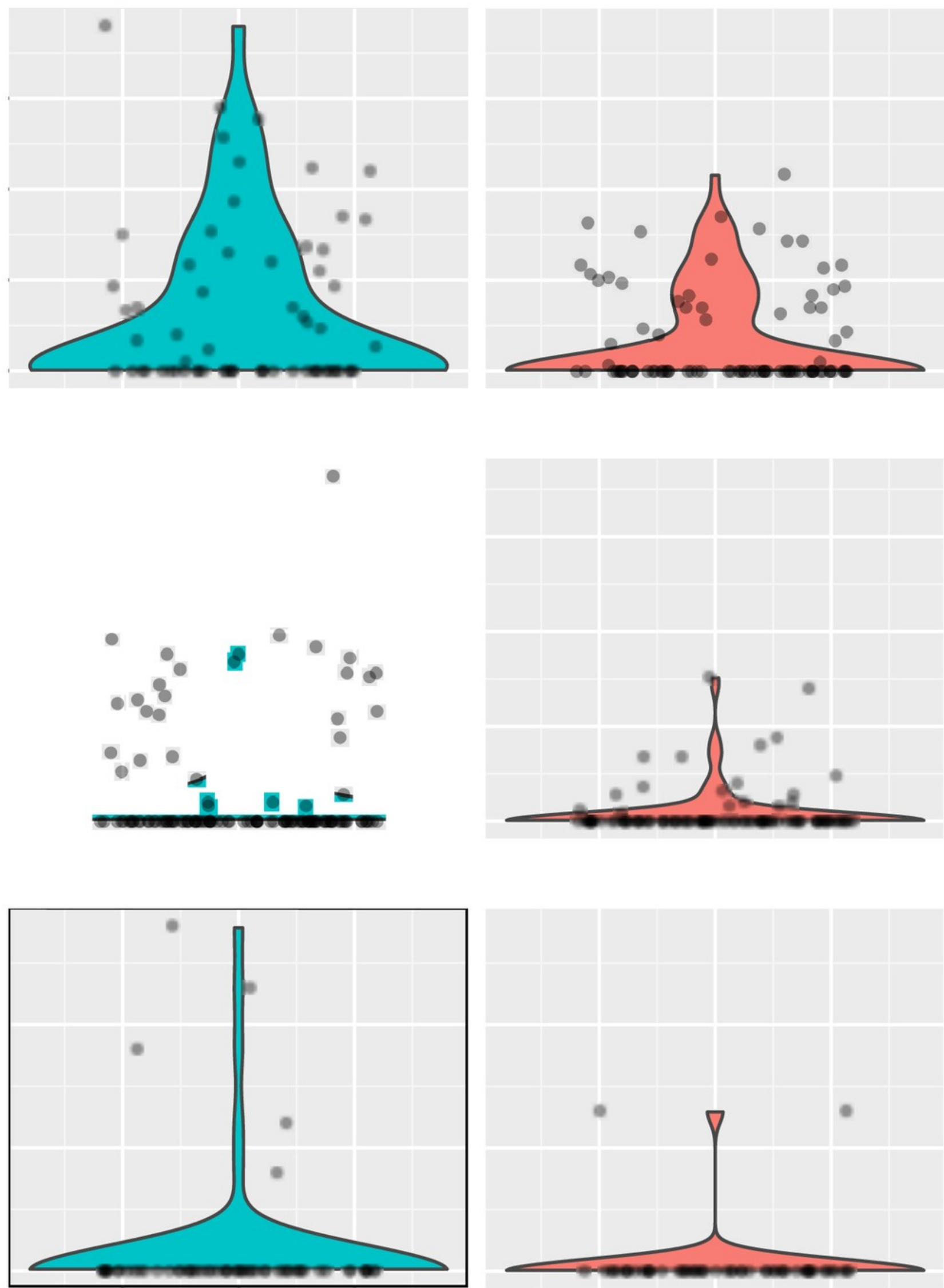


\section{Figure 2 (on next page)}

Ovaries by treatment and size.

Alternate image. Treatment with $n$-pentacosane $\left(C_{25}\right)$ reduced the number of oocytes in the ovaries of queenless workers, and there was a strong positive effect of worker body size (note different $y$ axes). The effect of $\mathrm{C}_{25}$ did not differ significantly between Large, Medium and Small workers. The violin plots show the kernel density estimate (i.e. estimated frequency: wide areas contain more data), and the scatter plot shows the raw data. The $x$ coordinates of the raw data are arbitrary: the points were randomly "jittered" horizontally to make overlapping points visible. 


\section{PeerJReviewing Manuscript}
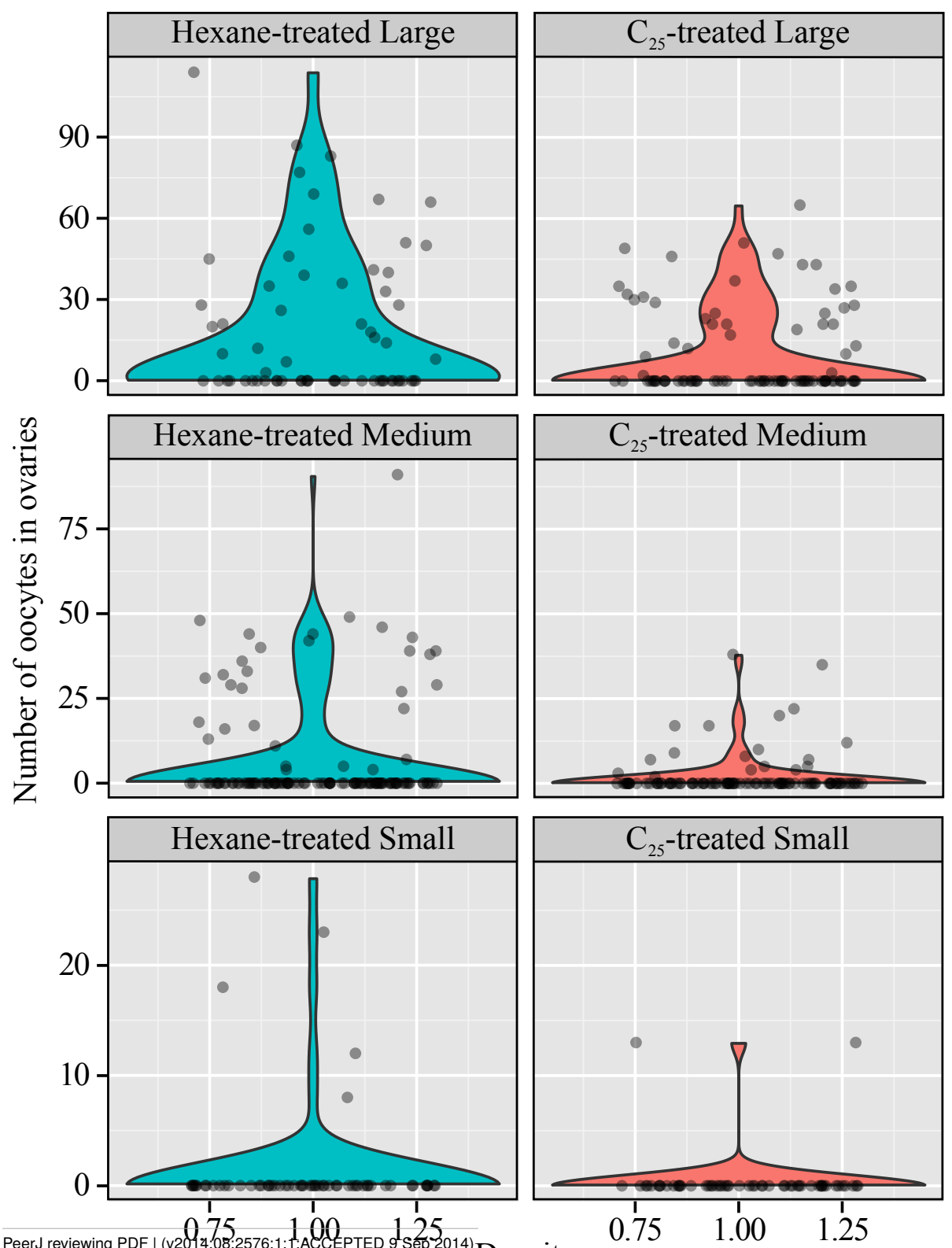

PeerJ reviewing PDF I (v2014:08:2576:1:1:ACCEPTED 9 Sep 2014) Density 